\title{
Confined Non-Newtonian Fluid Flow and Heat and Mass Transfers Induced by both Thermal and Solutal Buoyancy Forces of Equal Intensities
}

\author{
T. Makayssi ${ }^{1}$, M. Naïmi ${ }^{1, *}$, M. Lamsaadi ${ }^{2}$, M. Hasnaoui ${ }^{3}$, A. Raji ${ }^{1}$ and A.Bahlaoui ${ }^{2}$ \\ ${ }^{I}$ Sultan Moulay Slimane University, Faculty of Sciences and Technologies, Physics Department, Laboratory of flows \\ and Transfers Modeling (LAMET), B.P. 523, Beni-Mellal, Morocco \\ ${ }^{2}$ Sultan Moulay Slimane University, Polydisciplinary Faculty, Physics Department,Interdisciplinary Laboratory of \\ Research in Sciences and Technologies (LIRST), B.P. 523, Beni-Mellal, Morocco \\ ${ }^{3}$ Cadi Ayyad University, Faculty of Sciences Semlalia, Physics Department, Laboratory of Fluids Mechanics and \\ Energetics (LMFE), B.P. 2390, Marrakech, Morocco
}

*Email Corresponding Author: naimi@fstbm.ac.ma,naimima@yahoo.fr

(Received February 11, 2009; accepted November 11, 2009)

\begin{abstract}
In this paper is reported an analytical and numerical study on double-diffusive natural convection in a non-Newtonian power-law fluid confined in a shallow horizontal rectangular enclosure submitted to uniform heat and mass fluxes along its short vertical sides, while the horizontal ones are insulated and impermeable. Here, the cases of aiding and opposing thermal and solutal buoyancy forces of equal intensities are considered. In the first part of the work the full governing equations are solved and the effects of the power-law behavior index, $n$, and the generalized thermal Rayleigh number, $R a_{T}$, are examined and analyzed. In the second part, an analytical solution, based on the parallel flow approximation valid in the case of a shallow cavity, is proposed and an excellent agreement of results between the two approaches is observed, which validates them mutually.
\end{abstract}

Keywords: Double-diffusive natural convection; Heat and mass transfers; Non-Newtonian fluids; Rectangular shallow enclosures

\section{NOMENCLATURE}

A aspect ratio of the enclosure

c value relative to the centre of the enclosure $(x, y)=(A / 2,1 / 2)$

$C_{S}$ dimensionless concentration gradient in the $x$-direction

$C_{T}$ dimensionless temperature gradient in the $x$ direction

D mass diffusivity

g acceleration due to gravity

$H^{\prime}$ height of the enclosure

$j^{\prime} \quad$ consant mass flux per unit area

$k \quad$ consistency index for a power-law fluid at the reference temperature

Le Lewis number

$L^{\prime} \quad$ length of the enclosure

$N$ buoyancy ratio

$n$ flow behavior index for a power-law fluid at the reference temperature

$\mathrm{Nu}$ local Nusselt number
$\overline{\mathrm{Nu}}$ mean Nusselt number

$\mathrm{Pr}$ generalized Prandtl number

$q^{\prime} \quad$ constant heat flux per unit area

$R a_{T}$ generalized thermal Rayleigh number

$S$ dimensionless concentration, $\left(=\left(S^{\prime}-S_{c}^{\prime}\right) / \Delta S^{*}\right)$

$S_{c}^{\prime}$ reference concentration at the geometric center of the enclosure

Sh local Sherwood number

$\overline{S h}$ mean Sherwood number

$\Delta S^{*}$ characteristic concentration,$\left(=j^{\prime} H^{\prime} / D\right)$

$T$ dimensionless temperature, $\left(=\left(T^{\prime}-T_{c}^{\prime}\right) / \Delta T^{*}\right)$

$T_{c}^{\prime} \quad$ reference temperature

$\Delta T^{*}$ characteristic temperature, $\left(=q^{\prime} H^{\prime} / \lambda\right)$ 
$(u, v)$ dimensionless axial and vertical velocities, $\left(=\left(u^{\prime}, v^{\prime}\right) /\left(\alpha / H^{\prime}\right)\right)$

$(x, y)$ dimensionless axial and vertical coordinates, $\left(=\left(x^{\prime}, y^{\prime}\right) / H^{\prime}\right)$

$\alpha \quad$ thermal diffusivity at the reference temperature

$\beta \quad$ thermal expansion coefficient at the reference temperature

$\lambda$ thermal conductivity at the reference temperature

\section{INTRODUCTION}

Double-diffusive, or thermosolutal, natural convection is a fluid motion due to simultaneous variations of temperature and concentration in the gravity field. Because of coupling between the fluid velocity and the diffusive (thermal and solutal) fields, double-diffusive convection is more complex than the convective flow which is associated with a single diffusive scalar, and many different behaviors may be expected. Such thermosolutal processes occur in several fields, including chemical engineering (deposition of thin films, roll-over in storage tanks containing liquefied natural gas, solution mining of salt caverns for crude oil storage), solid-state physics (solidification of binary alloy and crystal growth), oceanography (melting and cooling near ice surfaces, sea water intrusion into freshwater lakes and the formation of layered or columnar structures during crystallization of igneous intrusions in the Earth's crust), geophysics (dispersion of dissolvent materials or particulate matter in flows), etc. In order to control these processes, a clear understanding of the nature of interaction between thermal and solutal buoyancy forces is necessary. For a review of the fundamental works in this area see, for instance, Pop and Ingham (2001).

On the other hand, most of the investigations, dealing with double-diffusive convection, were focused on the case of a rectangular enclosure confining a Newtonian fluid. These can be classified under three types, according to the imposed thermal and solutal boundary conditions. In the first type, the cavity is subjected to a vertical solutal gradient and a horizontal thermal one (Kalla et al., 2001). In the second type, both the temperature and concentration gradients are imposed transversally (Benacer and Gobin 1996; Mamou et al. 2001). In the third type, as in the present case, both the thermal and solutal gradients are imposed laterally (Ouriemi et al., 2006).

As far as we know, there are very few investigations dealing with double-diffusion convection inside rectangular enclosures confining non-Newtonian fluids. Among them are those performed while considering a saturated porous medium (Getachew et al. 1998; Benhadji and Vasseur 2001). In the case of a clear fluid medium, the only one is that conducted lately by Makayssi et al. (2008). All these studies have examined analytically and numerically the effect of the flow behavior index, the Lewis number and the buoyancy ratio on convection heat and mass transfers in the situation where both thermal and solutal buoyancy driven forces act in the same direction (the aiding case).
$\mu \quad$ dynamic viscosity for a Newtonian fluid at the reference temperature
$\mu_{a}$ dimensionless viscosity function for a non- Newtonian power-law fluid
$\Omega \quad$ dimensionless vorticity, $\left(=\Omega^{\prime} /\left(\alpha / H^{\prime 2}\right)\right)$
$\psi \quad$ dimensionless stream function $\left(=\psi^{\prime} / \alpha\right)$
$\rho$ density of fluid at the reference temperature dimensional variables

Non-Newtonian flows are of importance and very present in many industrial processes such as paper making, oil drilling, slurry transporting, food processing, polymer engineering and many others. Some of these processes are discussed by Jaluria (2003).

In order to contribute to fill the gap left by the lack of studies on the area, at least partly, the present investigation deals with natural convection heat and mass transfers inside a two-dimensional horizontal rectangular enclosure, filled with a non-Newtonian power-law fluid. The cavity is submitted to uniform heat and mass fluxes from the short vertical sides, while the long horizontal boundaries are insulated and impermeable. The study is particularly focused on the cases where thermal and solutal buoyancy driven forces can act in the same or opposite directions, but with the same intensities $(|N|=1$, where $N$ is the buoyancy ratio defined below). The general case (i.e. that where the driven buoyancy forces have not necessarily the same intensities) has been examined, while considering Newtonian fluids, by Ouriemi et al. (2006), for a clear fluid-filled cavity. These authors have emphasized the case $N=-1$ but without comparing with the case $N=1$.

In the following, the mathematical model and the solution procedure are discussed. The results presented here, for $N=-1$ and $N=1$, are relevant to a better understanding of double diffusive convection in clear non-Newtonian fluids media.

\section{MATHEMATICAL FORMULATiON}

\subsection{Problem Statement}

The studied configuration, which is sketched in Fig. 1, is a rectangular enclosure of height $H^{\prime}$ and length $L^{\prime}$ with the long horizontal rigid walls insulated and impermeable and the short vertical ones submitted to constant heat and mass densities of fluxes, $q^{\prime}$ and $j^{\prime}$, respectively. For this problem, thermal and solutal buoyancy driven forces can act in the same or opposite directions with the same intensities. The nonNewtonian fluids considered here are those for which the rheological behavior can be described by the powerlaw model, proposed by Ostwald-De Waele, whose expression, in terms of laminar viscosity function, is

$\mu_{a}^{\prime}=k\left[2\left(\left(\frac{\partial u^{\prime}}{\partial x^{\prime}}\right)^{2}+\left(\frac{\partial v^{\prime}}{\partial y^{\prime}}\right)^{2}\right)+\left(\frac{\partial u^{\prime}}{\partial y^{\prime}}+\frac{\partial v^{\prime}}{\partial x^{\prime}}\right)^{2}\right]^{\frac{n-1}{2}}$ 
where $n$ is the power-law index and $k$ is an empirical coefficient known as the consistency factor, which is an indicator of the degree of fluids viscosity. Note that for $n=1$ the power-law model reduces to the Newton's law by setting $k=\mu$. Thus, the deviation of $n$ from unity characterizes the degree of non-Newtonian behavior of the fluid. Specifically, when $n$ is in the range $0<n<1$ the fluid is said to be pseudo-plastic (or shear-thinning) and the viscosity function is found to decrease by increasing the shear rate. On the other hand when $n>1$ the fluid is said to be dilatant (or shearthickening) and the viscosity function increases by increasing the shear rate. Dilatant fluids are generally much less frequent than pseudo-plastic ones. Though the Ostwald-de Wale model does not converge to a Newtonian behavior in the limit of zero and maximum shear rates, it presents however the advantage to be simple and mathematically tractable. In addition, the rheological behavior of many substances can be adequately represented by this model for relatively large range of shear rates (or shear stresses) making it useful, at least for engineering purposes, and justifying its use in most theoretical investigations of fluids having pseudo-plastic or dilatant behaviors. On the other hand, the main assumptions made here are those commonly used, i.e., the flow is laminar and twodimensional (Siginer and Valenzuela-Rendon, 2000), the viscous dissipation is negligible, the interactions between heat and mass exchanges, known under the name of Soret and Duffour effects, are negligible, the fluid is incompressible and its physical properties are considered temperature independent except the density in the buoyancy term which obeys the Boussinesq approximation.

\subsection{Governing Equations and Boundary Conditions}

On the basis of what precedes, the dimensionless governing equations, written in terms of vorticity, $\Omega$, temperature, $T$, concentration, $S$, and stream function, $\psi$, are:

$$
\begin{aligned}
& \frac{\partial \Omega}{\partial t}+\frac{\partial(u \Omega)}{\partial x}+\frac{\partial(v \Omega)}{\partial y}=\operatorname{Pr}\left[\mu_{a} \nabla^{2} \Omega+2 \vec{\nabla} \mu_{a} \cdot \vec{\nabla} \Omega\right]+S_{\Omega} \\
& \frac{\partial T}{\partial t}+\frac{\partial(u T)}{\partial x}+\frac{\partial(v T)}{\partial y}=\nabla^{2} T \\
& \frac{\partial S}{\partial t}+\frac{\partial(u S)}{\partial x}+\frac{\partial(v S)}{\partial y}=\frac{1}{L e} \nabla^{2} S \\
& \nabla^{2} \psi=-\Omega
\end{aligned}
$$

where

$$
u=\frac{\partial \psi}{\partial y} ; v=-\frac{\partial \psi}{\partial x} ; \Omega=\frac{\partial v}{\partial x}-\frac{\partial u}{\partial y}
$$$$
\mu_{a}=\left[2\left(\left(\frac{\partial u}{\partial x}\right)^{2}+\left(\frac{\partial v}{\partial y}\right)^{2}\right)+\left(\frac{\partial u}{\partial y}+\frac{\partial v}{\partial x}\right)^{2}\right]^{\frac{n-1}{2}}
$$

and

$$
S_{\Omega}=\operatorname{Pr}\left[\left(\frac{\partial^{2} \mu_{a}}{\partial x}-\frac{\partial^{2} \mu_{a}}{\partial y}\right)\left(\frac{\partial u}{\partial y}+\frac{\partial v}{\partial x}\right)\right.
$$

$$
\left.-2 \frac{\partial^{2} \mu_{a}}{\partial x \partial y}\left(\frac{\partial u}{\partial x}-\frac{\partial v}{\partial y}\right)+R a_{T}\left(\frac{\partial T}{\partial x}+N \frac{\partial S}{\partial y}\right)\right]
$$

The dimensionless boundary conditions, for the physical system considered here, are

$$
\begin{aligned}
& u=v=\psi=\frac{\partial T}{\partial x}+1=\frac{\partial S}{\partial x}+1=0 \text { for } x=0 \text { and } A \\
& u=v=\psi=\frac{\partial T}{\partial y}=\frac{\partial S}{\partial y}=0 \text { for } y=0 \text { and } 1
\end{aligned}
$$

As regards the vorticity, which is unknown at the boundaries, the woods relation (Roache, 1982) is used, for its accuracy and stability.

In addition to the power-law index, $n$, the present problem is governed by five other dimensionless parameters, namely, the aspect ratio of the enclosure, $A$, the Lewis number, $L e$, the buoyancy ratio, $N$, the generalized Prandtl, $P r$, and thermal Rayleigh, $R a_{T}$, numbers, the expressions of which are

$$
\begin{aligned}
& A=\frac{L^{\prime}}{H^{\prime}}, L e=\frac{\alpha}{D}, N=\frac{\beta_{S} \Delta S^{\prime}}{\beta_{T} \Delta T^{\prime}}, \operatorname{Pr}=\frac{(k / \rho) H^{\prime 2-2 n}}{\alpha^{2-n}} \\
& \text { and } R a_{T}=\frac{g \beta H^{\prime 2 n+2} q^{\prime}}{(k / \rho) \alpha^{n} \lambda}
\end{aligned}
$$

Notice that it is possible to recover the Newtonian expressions of $\operatorname{Pr}$ and $R a_{T}$ by, simply, setting $n=1$ and replacing $k$ by the Newtonian viscosity, $\mu$.

\section{NUMERICAL APPROACH}

The two-dimensional governing equations are solved by using the well known second order central finite difference method with a regular mesh size. The integration of Eqs. (2)-(4) is performed with the alternating-direction implicit method (ADI) (Peaceman and Rachford Jr., 1955). To satisfy the mass conservation, Eq. (5) is solved by a point successive over-relaxation method (PSOR) with an optimum relaxation factor calculated by the Franckel formula (Roache, 1982). The mesh size is chosen on the basis of a compromise between running time and accuracy of the results. The procedure is based on grid refinement until the numerical results agree with the parallel flow ones within reasonable accuracy. Hence, a uniform grid of $321 \times 81$ is selected for $A=24$ (value used for the numerical computations), and it is estimated sufficient to model accurately the flow, temperature and concentration fields within the cavity. To satisfy the continuity equation, the convergence criterion $\sum_{i, j}\left|\psi_{i, j}^{k+1}-\psi_{i, j}^{k}\right| / \sum_{i, j}\left|\psi_{i, j}^{k+1}\right|<10^{-4}$ is adopted, where $\psi_{i, j}^{k}$ is the value of the stream function at the $k$ th iteration level. The time step size, $\delta t$, is varied in the range $10^{-7} \leq \delta t \leq 10^{-4}$, depending on the values of the governing parameters. More precisely, the small values of $\delta t$ are used for high values of $n$ and $R a_{T}$. 
With the Ostwald power-law model, the dimensionless viscosity function, given by Eq. (7), tends towards infinity, for $0<n<1$, at the level of the cavity corners, where the velocity gradients tend towards zero, which renders impossible direct numerical computations. This difficulty is, however, overcome by using average values for the corner viscosity function making, thus, the computations possible and stable.

The local heat and mass transfers through the fluid layer filling the cavity can be expressed in terms of the local Nusselt and Sherwood numbers, respectively, defined as

$$
\begin{aligned}
& N u(y)=\frac{q^{\prime}}{\lambda \Delta T^{\prime} / L^{\prime}}=\frac{A}{\Delta T}=\frac{1}{\Delta T / A} \\
& \operatorname{Sh}(y)=\frac{j^{\prime}}{D \Delta S^{\prime} / L^{\prime}}=\frac{A}{\Delta S}=\frac{1}{\Delta S / A} \\
& \text { where } \\
& \qquad T=T(0, y)-T(A, y)
\end{aligned}
$$

$\Delta S=S(0, y)-S(A, y)$ are the side to side dimensionless local temperature and concentration differences, respectively. This definition is, however, notoriously inaccurate owing to the uncertainty of the temperature and concentration values evaluated at the two vertical walls (edge effects). Instead, the Nusselt and Sherwood numbers are calculated on the basis of a temperature and concentration differences between two vertical sections, far from the end sides. Thus, by analogy with Eqs. (12) and (13), and considering two infinitesimally close sections, the local Nusselt and Sherwood numbers can be defined, respectively, by:

$$
\begin{aligned}
& N u(y)=\lim _{\delta x \rightarrow 0} \delta x / \delta T=\lim _{\delta x \rightarrow 0} \frac{1}{\delta T / \delta x}=-\frac{1}{(\partial T / \partial x)_{x=A / 2}} \\
& S h(y)=\lim _{\delta x \rightarrow 0} \delta x / \delta S=\lim _{\delta x \rightarrow 0} \frac{1}{\delta S / \delta x}=-\frac{1}{(\partial S / \partial x)_{x=A / 2}}
\end{aligned}
$$

where $\delta x$ is the distance between two symmetrical sections with respect to the central one. The corresponding average Nusselt and Sherwood numbers are, respectively, calculated at different locations, as follows:

$$
\begin{aligned}
& \overline{N u}=\int_{0}^{1} N u(y) d y \\
& \overline{S h}=\int_{0}^{1} \operatorname{Sh}(y) d y
\end{aligned}
$$

As an additional check of the results accuracy, energy and matter balances are systematically verified for the system at each numerical code running. Thus, the overall heat and mass transfers, through each vertical plane, are evaluated and compared with the quantities of heat and mass furnished to the system at $x=0$. For the results reported here, the energy and matter balances are satisfied within $2 \%$ as a maximum difference.

Typical numerical results, in terms of streamlines, isotherms and isoconcentrations, are presented in Fig. 2, obtained, for $A=24, L e=100, R a_{T}=10^{5}$ and various values of $n$. As appears, from these figures, the flow is parallel to the horizontal boundaries of the enclosure and the temperature and the concentration are linearly stratified in the $x$-direction of the core region.
The approximate analytical solution, developed in the next section, relies on these observations.

\section{Parallel Flow Approach}

The results presented in Fig. 2, allow the following appropriate simplifications

$$
\begin{aligned}
u(x, y) & =u(y), v(x, y)=0, \psi(x, y)=\psi(y), \\
T(x, y) & =C_{T}(x-A / 2)+\theta_{T}(y) \\
S(x, y) & =C_{S}(x-A / 2)+\theta_{S}(y)
\end{aligned}
$$

where $C_{T}$ and $C_{S}$ are unknown constant temperature and concentration gradients in the $x$-direction. In such a situation the full non-dimensional governing equations reduce to:

$$
\begin{aligned}
& \frac{d^{2}}{d y^{2}}\left[\left|\frac{d u}{d y}\right|^{n-1} \frac{d u}{d y}\right]=\left(C_{T}+N C_{S}\right) R a_{T}=E R a_{T} \\
& C_{T} u=\frac{d^{2} \theta_{T}}{d y^{2}} \\
& \operatorname{LeC}_{S} u=\frac{d^{2} \theta_{S}}{d y^{2}}
\end{aligned}
$$

with

$$
\begin{aligned}
& u=\frac{d \theta_{T}}{d y}=\frac{d \theta_{S}}{d y}=0 \text { for } y=0 \text { and } 1 \\
& \int_{0}^{1} u(y) d y=0
\end{aligned}
$$

as boundary and return flow conditions, respectively.

The integration of Eqs. (19)-(21), coupled with the conditions (22) and (23), leads to analytical expressions of velocity, temperature and concentration. However, such an operation is difficult to carry out owing to the particular nature of the governing equations and requires, therefore, a special numerical treatment. In fact, the non-linearity of the fluid behavior and the change of $d u / d y$ sign, due to the return flow, impose that the velocity expressions are different depending on whether $0 \leq y \leq y_{0}, y_{0} \leq y \leq y_{1}$ or $y_{1} \leq y \leq 1$, where $y_{0}$ and $y_{1}=1-y_{0}$ (because of the centro-symmetry of the core flow) are the vertical coordinate values for which $d u / d y=0$. They are derived from Eq. (23), which is numerically solved by using a combination of the Regula-Falsi iteration method (Gourdin and Boumahrat, 1989) and the Gauss-Legendre integration method (Sibony and Mardon, 1982). To reduce the velocity, temperature and concentration expressions, the function $f(y)=\left(y^{2}-y+y_{0} y_{1}\right) / 2$ is introduced. Thus, for:

$$
\begin{aligned}
& -0 \leq y \leq y_{0} \\
& u(y)=E^{1 / n} \operatorname{Ra}_{T}^{1 / n}\left[\int_{0}^{y}[f(y)]^{1 / n} d y\right] \\
& \theta_{T}(y)=C_{T} E^{1 / n} \operatorname{Ra}_{T}^{1 / n}\left[\int_{0}^{y}\left[\int_{0}^{y}\left[\int_{0}^{y}[f(y)]^{1 / n} d y\right] d y\right] d y\right.
\end{aligned}
$$


T. Makayssi et al. / JAFM, Vol. 4, No. 1, pp. 97-106, 2011.

$$
+\theta_{T}(0)
$$

- $y_{0} \leq y \leq y_{1}$

$$
\begin{aligned}
u(y)= & E^{1 / n} R a_{T}^{1 / n}\left[\int_{0}^{y_{0}}[f(y)]^{1 / n} d y\right. \\
& +\int_{y}^{y_{0}}[-f(y)]^{1 / n} d y \\
\theta_{T}(y)= & C_{T} E^{1 / n} \operatorname{Ra}_{T}^{1 / n}\left[\frac{\left(y-y_{0}\right)^{2}}{2} \int_{0}^{y_{0}}[f(y)]^{1 / n} d y\right. \\
& +\int_{y_{0}}^{y}\left[\int_{y_{0}}^{y}\left[\int_{y}^{y}[-f(y)]^{1 / n} d y\right] d y\right] d y \\
& +\left(y-y_{0}^{y_{0}} \int_{0}^{y_{0}}\left[\int_{0}^{y}[f(y)]^{1 / n} d y\right] d y\right. \\
& \left.+\int_{0}^{y_{0}}\left[\int_{0}^{y}\left[\int_{0}^{y}[f(y)]^{1 / n} d y\right] d y\right] d y\right] \\
& +\theta_{T}(0)
\end{aligned}
$$

- $y_{1} \leq y \leq 1$

$$
\begin{aligned}
u(y)= & E^{1 / n} R a_{T}^{1 / n}\left[\int_{0}^{y_{0}}[f(y)]^{1 / n} d y\right. \\
& \left.+\int_{y_{1}}^{y_{0}}[-f(y)]^{1 / n} d y+\int_{y_{1}}^{y}[f(y)]^{1 / n} d y\right] \\
\theta_{T}(y)= & C_{T} E^{1 / n} R a_{T}^{1 / n}\left[\frac{1}{2}\left(y-y_{1}\right)\left(y+y_{1}-2\right)\right. \\
& \times\left[\int_{0}^{y_{0}}[f(y)]^{1 / n} d y+\int_{y_{1}}^{y_{0}}[-f(y)]^{1 / n} d y\right] \\
& +\int_{y_{1}}^{y}\left[\int_{1}^{y}\left[\int_{y_{1}}^{y}[f(y)]^{1 / n} d y\right] d y\right] d y \\
& +\int_{y_{0}}^{y_{1}}\left[\int_{y_{0}}^{y}\left[\int_{y}^{y_{0}}[-f(y)]^{1 / n} d y\right] d y\right] d y \\
& +\frac{1}{2}\left(y_{1}-y_{0}\right)^{2} \int_{0}^{y_{0}}[f(y)]^{1 / n} d y \\
& \left(y_{1}-y_{0} \int_{0}^{y_{0}}\left[\int_{0}^{y}[f(y)]^{1 / n} d y\right] d y\right. \\
&
\end{aligned}
$$

$$
\begin{aligned}
& \left.+\int_{0}^{y_{0}}\left[\int_{0}^{y}\left[\int_{0}^{y}[f(y)]^{1 / n} d y\right] d y\right] d y\right] \\
& +\theta_{T}(0)
\end{aligned}
$$

The expression of $\theta_{S}(y)$, given by

$$
\theta_{S}(y)=L e \frac{C_{S}}{C_{T}} \theta_{T}(y)
$$

is obtained by eliminating $u$ from Eqs. (20) and (21), and integrating twice the resulting equation taking into account of Eq. (22) and the centro-symmetry of thermal and solutal fields in the core region. The exploitation of such a property and the use of Eq. (30) give, respectively:

$$
\begin{aligned}
\theta_{T}(0)= & -C_{T} E^{1 / n} R a_{T}^{1 / n}\left[\frac{\left(1 / 2-y_{0}\right)^{2}}{2} \int_{0}^{y_{0}}[f(y)]^{1 / n} d y\right. \\
& +\int_{y_{0}}^{1 / 2}\left[\int_{y_{0}}^{y}\left[\int_{y}^{y_{0}}[-f(y)]^{1 / n} d y\right] d y\right] d y \\
& +\left(1 / 2-y_{0}\right) \int_{0}^{y_{0}}\left[\int_{0}^{y}[f(y)]^{1 / n} d y\right] d y \\
& \left.+\int_{0}^{y_{0}}\left[\int_{0}^{y}\left[\int_{0}^{y}[f(y)]^{1 / n} d y\right] d y\right] d y\right]
\end{aligned}
$$

and

$$
\theta_{S}(0)=L e \frac{C_{S}}{C_{T}} \theta_{T}(0)
$$

The expression of $\psi(y)$ can be deduced from that of $u(y)$ by integration of Eq. (6) taking into account of Eq. (10). Therefore, the stream function at the center of the enclosure, which is a measure of the flow intensity, can be expressed by

$$
\begin{aligned}
\psi_{\mathrm{c}} & =\psi(A / 2,1 / 2)=E^{1 / n} \operatorname{Ra}_{T}^{1 / n}\left[\left(1 / 2-y_{0}\right)\right. \\
& \times \int_{0}^{y_{0}}[f(y)]^{1 / n} d y+\int_{0}^{y_{0}}\left[\int_{0}^{y}[f(y)]^{1 / n} d y\right] d y \\
& \left.+\int_{y_{0}}^{1 / 2}\left[\int_{y}^{y_{0}}[-f(y)]^{1 / n} d y\right] d y\right]
\end{aligned}
$$

On the other hand, $C_{T}$ and $C_{S}$ are evaluated from thermal and solutal boundary conditions imposed on the end walls. Because of the turning flow at the end regions of the fluid layer, the boundary conditions in the $x$-direction, Eq. (9), could not be satisfied by the parallel flow approximation. Instead, the expressions of $C_{T}$ and $C_{S}$ are determined by matching the core solution, Eq. (15), to the integral solution for the end regions, which consists on the integration of Eqs. (3) and (4), together with the boundary conditions (9) and (10), by considering the arbitrary control volume of Fig. 1. This yields: 


$$
\begin{aligned}
C_{T}+1 & =\int_{0}^{1} u(y) \theta_{T}(y) d y \\
C_{S}+1 & =\underset{0}{\operatorname{Le}} \int_{0}^{1} u(y) \theta_{S}(y) d y
\end{aligned}
$$

Moreover, the substitution of $u(y), \theta_{T}(y)$ and $\theta_{S}(y)$ expressions to Eqs. (34) and (35) gives:

$$
C_{T}=\frac{1}{A_{n} E^{2 / n} R a_{T}^{2 / n}-1}
$$

and

$$
C_{S}=\frac{1}{A_{n} L e^{2} E^{2 / n} R a_{T}^{2 / n}-1}
$$

where the coefficient $A_{n}$, which depends only on $n$, is calculated with the Gauss-Legendre integration method. In addition, knowing that $E=C_{T}+N C_{S}$, the transcendental equation

$$
\begin{aligned}
& L e^{2} A_{n}^{2} R a_{T}^{4 / n} E^{1+4 / n}-A_{n}\left(1+L e^{2}\right) R a_{T}^{2 / n} E^{1+2 / n}- \\
& \left(L e^{2}+N\right) A_{n} R a_{T}^{2 / n} E^{2 / n}+E+(N+1)=0
\end{aligned}
$$

is obtained and solved by the Regula-Falsi iteration method, for each given value of $L e, n, N$ and $R a_{T}$, which leads to the value of $E$ and those of $C_{T}$ and $C_{S}$, from Eqs. (36) and (37), respectively.

Finally, taking into account of Eqs. (14)-(17), (36) and (37) the Nusselt and Sherwood numbers are constant and can be, respectively, expressed as

$$
\begin{aligned}
& N u=-1 / C_{T}=1-A_{n} E^{2 / n} R a_{T}^{2 / n}=\overline{N u} \\
& S h=-1 / C_{S}=1-A_{n} L e^{2} E^{2 / n} R a_{T}^{2 / n}=\overline{S h}
\end{aligned}
$$

\section{RESULTS AND DISCUSSION}

With boundary conditions of Newman type, i.e. imposed uniform heat and mass fluxes like in our case, the flow, heat and mass transfer properties become independent of the large values of the cavity aspect ratio, $A$. The parallel flow solution, developed in the previous section, is thus valid asymptotically in the limit of a shallow cavity $(A>>1)$. Therefore, numerical tests are performed to determine the smallest value of $A$ leading to results reasonably close to those of large aspect ratio approximation. On the other hand, for the non-Newtonian fluids considered here, the Prandtl number, $P r$, is large enough such that the convection becomes insensitive to any change of the large values of this parameter (Lamsaadi et al., 2006). On the basis of this, the simulations are conducted with $\mathrm{Pr} \rightarrow \infty$, i.e. by neglecting the convective terms on the left hand side of Eq. (2).

In addition to that, the Lewis number, $L e$, which does not contain mechanical properties related to a flow since it compares the relative importance of thermal and molecular diffusions, should not be very different from that of Newtonian liquids, although molecular diffusion, even apparent, is more or less inhibited in liquids extremely viscous (Guyon et al., 2001). Therefore, a magnitude order of 100 seems appropriate (York et al.,
2007) compared to 1 which is unrealistic even though it is often used in numerical simulations.

Hence, the natural double-diffusive convection flow developed inside the enclosure is governed by the buoyancy ratio, $N$ (which can takes 1 or -1 as values, in order to simulate thermal and solutal buoyancy forces acting in the same or opposite directions with the same intensities), the power-law index, $n$ (which is varied, in this study, from 0.6 to 1.4 to include shear-thinning $(0<n<1)$, Newtonian $(n=1)$ and shear-thickening $(n>1)$ fluids), and the thermal Rayleigh number, $R a_{T}$ . It is necessary to point out that, unlike the case where the cavity is heated and salted from below, for which the convection appears when a certain threshold of $R a_{T}$ is crossed (Mamou et al., 2001), such a phenomenon starts from any small value of $R a_{T}$ in the present case.

\subsection{Validation of the Approximate Parallel Flow Analytical Solution}

The inspection of the streamlines (Fig. 2), isotherms (Fig. 3) and isoconcentrations (Fig. 4), allows affirming the existence of an analytical solution, for the present problem, owing to the parallelism and the stratification aspects that flow, temperature and concentration fields exhibit, respectively, in the major part of the enclosure, except near the side edges. Moreover, Fig. 5, comparing the corresponding horizontal velocity (a), temperature (b) and concentration (c) profiles, obtained analytically (continuous and dashed lines) and numerically (empty and full circles) at mid-length of the cavity, along the vertical direction, testifies of the excellent agreement between the two types of solutions, which validates the parallel flow hypothesis.

\subsection{Effect of the Flow Behavior Index}

Useful information, concerning the influence of the non-Newtonian rheological behavior (i.e. the effect of $n$ ) on the flow, thermal and solutal fields, can be obtained from the examination of Figs. 2-4. Thus, the unicellular nature of the flow is always preserved for $n$ varying in its range. In addition, outside the end regions (flow zones adjacent to the vertical sides), whose length reduces with an increasing $n$, the flow, and the temperature and concentration fields are respectively parallel to the long walls and linearly stratified in the horizontal direction, whatever the value of $n$. These observations allow important simplifications which are used in the section 4 to transform the governing partial differential equations to ordinary ones.

On the other hand, qualitatively, the isotherms and isoconcentrations seem to be more sensitive to the variations of $n$, than the streamlines, since they tend to be flat (i.e. horizontal) with a decreasing $n$. These qualitative observations are consistent with the horizontal velocity (top), temperature (middle) and concentration (bottom) profiles, which are presented in Fig. 5. Indeed, according to Eq. (7), small values of $n$ imply lower viscosity levels, and thus stronger flow and hence more mixing effect, which leads to flatter temperature and concentration profiles. 
It follows from the preceding discussion that, compared to Newtonian case $(n=1)$, the shear-thinning behavior $(0<n<1)$ enhances the convection whereas the shearthickening one $(n>1)$ reduces it. Besides, Fig. 6 gives a good confirmation of such a tendency for a wide range of the thermal Rayleigh number.

\subsection{Effect of the Thermal Rayleigh Number}

The effect of the thermal Rayleigh number, $R a_{T}$, on the flow intensity $\left(\left|\psi_{c}\right|\right.$, top) and heat $(\overline{N u}$, middle $)$ and mass ( $\overline{S h}$, bottom) transfers rates is illustrated on Fig. 6. When $R a_{T}$ is small enough $\left(R a_{T}<20\right)$, convection heat and mass transfers is essentially ensured by pseudo-diffusion since $0.02<\left|\psi_{c}\right|<0.07$,

$\overline{N u} \approx 1$ and $2<\overline{S h}<20$. Outside this zone, an increase of $R a_{T}$ leads to an increase of $\left|\psi_{c}\right|$ and $\overline{S h}$ without affecting first $\overline{N u}$, which exhibits real increase only when $R a_{T}>100$, dependently on $n$. Otherwise, for the high values of $R a_{T}$, all these quantities increase in a monotonous way, due to the obvious contribution of buoyancy effects in promoting the convection heat and mass transfers. We also note that, owing to the fact that $L e=100$, the mass transfer is more pronounced than the heat transfer.

\subsection{Comparison between Results Related to Aiding and Opposing Cases}

In order to show how the direction of thermal and solutal buoyancy forces can affect convection heat and mass transfers, especially when these forces act with the same intensities, some results related to flow, thermal and solutal fields and their intensities are presented for two values of the buoyancy ratio: $N=-1$ and $N=1$. Hence, as can be seen from Figs. 2-6, which compare the two situations, there is almost no difference between the results corresponding to the opposing $(N=-1)$ and cooperating $(N=1)$ cases. This can be attributed to the fact that with a large value of Lewis number, like that used in the present study ( $L e=100)$, the axial concentration gradient is negligible when compared to the axial temperature one $\left(\left|C_{S}\right|<<\left|C_{T}\right|\right)$, as shown in Table 1, leading to $E \approx C_{T}$, which explains why the results are almost the same for the two cases.

\section{Conclusion}

This study deals, numerically and analytically, with natural double-diffusive convection in a twodimensional horizontal shallow enclosure, filled with non-Newtonian power-law fluids, in the case where both short vertical sides are submitted to uniform heat and mass fluxes while the horizontal boundaries are insulated and impermeable. Here the thermal and solutal buoyancy driving forces are, particularly, considered as acting in the same or opposite directions with the same intensities. The following points summarize the main conclusions of the present investigation:

- The approximate analytical solution, developed on the basis of the parallel flow hypothesis in the core region of the cavity, is found to agree perfectly with the numerical solution, obtained by solving numerically the full governing equations in the chosen ranges of the governing parameters.

- The fluid flow and heat and mass transfers' properties seem to be rather sensitive to the flow behavior index, $n$. Thus, compared to Newtonian case $(n=1)$, the shearthinning behavior $(0.6<n<1)$ enhances the convection heat and mass transfers while the shearthickening one $(1<n<1.4)$ leads to the opposite effect.

- The influence of the thermal Rayleigh number, $R a_{T}$, is such that in general, for $R a_{T}>100$, any increase of this parameter enhances convection heat and mass transfers.

Because of the large value of Lewis number ( $L e=100$ ), convection heat and mass transfers remain almost unaffected by the direction of thermal and solutal buoyancy driving forces when they are of identical intensities $(|N|=1)$.

\section{REFERENCES}

Benacer, R. and D. Gobin (1996). Cooperating thermosolutal convection in enclosures I. Scale analysis and mass transfer, International Journal of Heat and Mass Transfer 39, 2671-2681.

Benhadji, K. and P. Vasseur (2001). Double diffusive convection in a shallow porous cavity filled with a non-Newtonian fluid. International Communications in Heat and Mass Transfer 28, 763-772.

Getachew, D., D. Poulikakos and W.J. Minkowycz (1998). Double-diffusive in a porous cavity saturated with non-Newtonian Fluid. Journal of Thermophysics and Heat Transfer 12, 437-446.

Gourdin, A. and M. Boumahrat (1989). Méthodes numériques appliquées. Paris: Lavoisier.

Guyon, E, J.-P. Hulin, and L. Petit (2001). Hydrodynamique Physique, Paris, France: EDP Sciences.

Jaluria, Y. (2003). Thermal processing of materials: From basic research to engineering. Journal of Heat Transfer 125, 957-979.

Kalla, L., P. Vasseur, R. Benacer, H. Beji and R. Duval (2001). Double-diffusive convection within a horizontal porous layer salted from the bottom and heated horizontally. International Communications in Heat and Mass Transfer 28, 1-10.

Lamsaadi, M., M. Naïmi and M. Hasnaoui (2006). Natural convection heat transfer in shallow 
T. Makayssi et al. / JAFM, Vol. 4, No. 1, pp. 97-106, 2011.

horizontal rectangular enclosures uniformly heated from the side and filled with non-Newtonian power-law fluids, Energy conversion and Management 47, 2535-2551.

Makayssi, T., M. Lamsaadi,, M. Naïmi, M. Hasnaoui, A. Raji and A. Bahlaoui (2008). Natural doublediffusive convection in a shallow horizontal rectangular cavity uniformly heated and salted from the side and filled with non-Newtonian power-law fluids: the cooperating case, Energy conversion and Management 49, 2016-2025.

Mamou, M., P. Vasseur and M. Hasnaoui (2001). On numerical stability analysis of double-diffusive convection in confined enclosures, Journal of Fluid Mechanics 433, 209-250.

Ouriemi, M., P. Vasseur, A. Bahloul and L. Robillard (2006). Natural convection in a horizontal layer of a binary mixture, International Journal of Thermal Sciences 45, 752-759.

Peaceman, D.W. and H.H. Rachford Jr. (1955). The numerical solution of parabolic and elliptic differential equations, Journal of the Society for Industrial and Applied Mathematics 3, 28-41.

Pop, I. and D.B. Ingham (2001). Convective Heat Transfer: Mathematical and Computational Modeling of Viscous Fluids and Porous Media, Oxford, UK: Elsevier Science \& Technology Books (Pergamon).

Roache, P.J. (1982). Computational fluid dynamics. Hermosa Publishers, Albuquerque, New Mexico.

Sibony, M. and J.-CI. Mardon (1982). Approximation et équations différentielles. Paris: Hermann.

Siginer, D.A. and A. Valenzuela-Rendon (2000). On the laminar free convection and stability of grade fluids in enclosures. International Journal of Heat and Mass Transfer 43, 3391-405.

York, P., U.P. Kompella, and B.Y. Shekunov (2007). Supercritical Fluid Technology for Drug Product Development, Drugs and the Pharmaceutical Sciences, Vol. 138, Oxfordshire, UK: Taylor \& Francis (Informa Healthcare).

Table 1 Comparison between $\boldsymbol{C}_{\boldsymbol{T}}, \boldsymbol{C}_{\boldsymbol{S}}$ and $E$ values obtained in the opposing and cooperating cases, for

$$
R a_{T}=10^{5}
$$

\begin{tabular}{|c|c|c|c|c|c|c|}
\hline \multirow[t]{2}{*}{$n \downarrow / N \rightarrow$} & \multicolumn{2}{|c|}{$C_{T}$} & \multicolumn{2}{|c|}{$C_{S}$} & \multicolumn{2}{|c|}{$E$} \\
\hline & -1 & 1 & -1 & 1 & -1 & 1 \\
\hline 0.6 & -0.006935 & -0.006934 & $-6.98 \times 10^{-7}$ & $-6.98 \times 10^{-7}$ & -0.006935 & -0.006935 \\
\hline 1.0 & -0.032721 & -0.032722 & $-3.38 \times 10^{-6}$ & $-3.38 \times 10^{-6}$ & -0.032722 & -0.032722 \\
\hline 1.4 & -0.103301 & -0.103299 & $-1.15 \times 10^{-5}$ & $-1.15 \times 10^{-5}$ & -0.103300 & -0.103300 \\
\hline
\end{tabular}

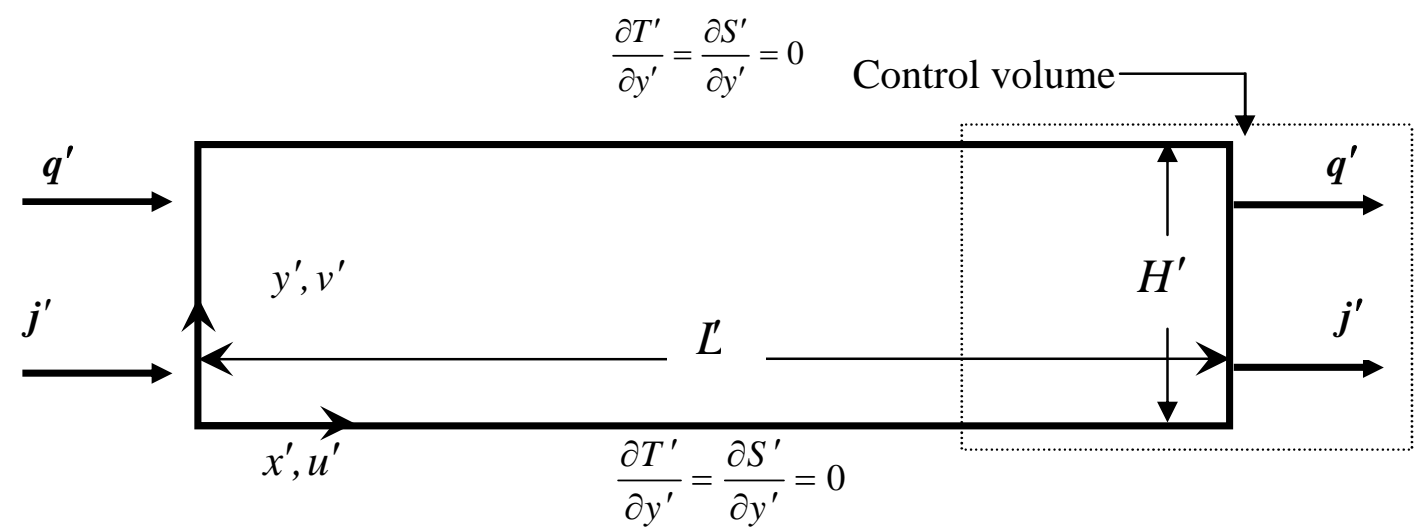

Fig. 1. Sketch of the geometry and coordinates system. 


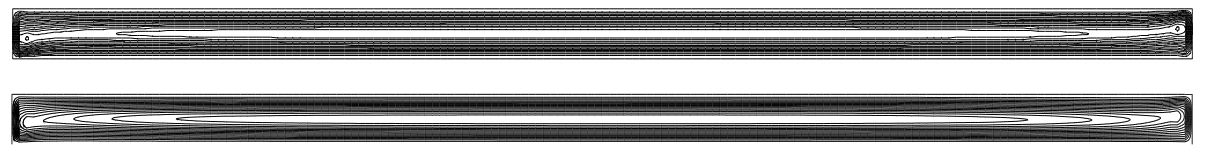

(a)

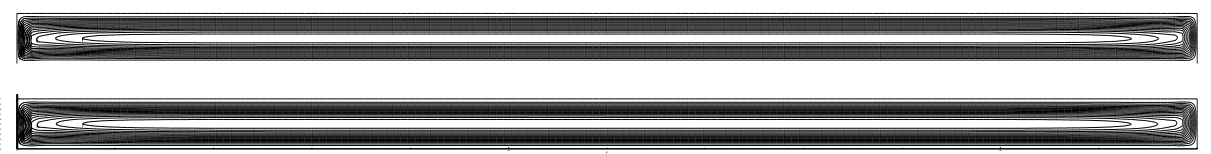

(b)

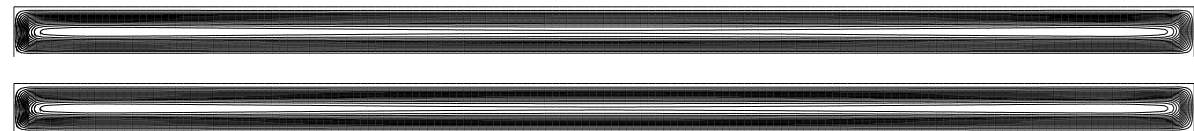

(c)

Fig. 2. Streamlines for $N=-\mathbf{1}$ (top), $N=\mathbf{1}$ (bottom), $A=\mathbf{2 4}, \boldsymbol{L e}=\mathbf{1 0 0}, \boldsymbol{R} \boldsymbol{a}_{T}=\mathbf{1 0} \mathbf{5}^{\mathbf{2}}$ and various values of $n$ : (a) $n=0.6$, (b) $n=1.0$ and (c) $n=1.4$.

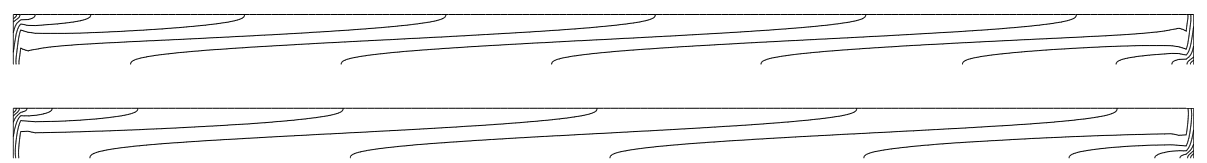

(a)

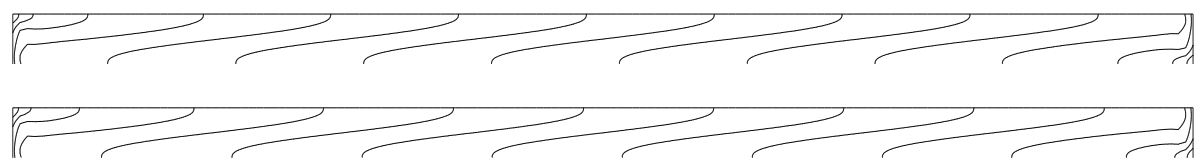

(b)

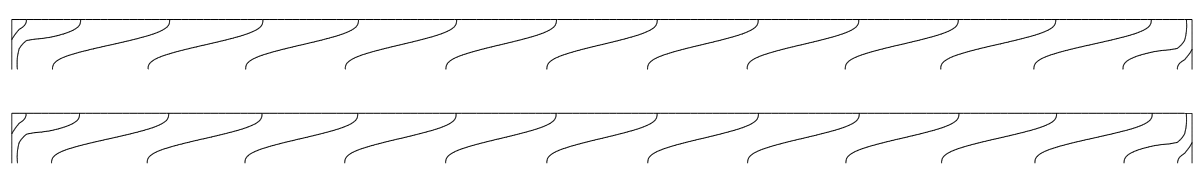

(c)

Fig. 3. Isotherms for $N=-1$ (top), $N=1$ (bottom), $A=\mathbf{2 4}, \boldsymbol{L e}=\mathbf{1 0 0}, \boldsymbol{R} \boldsymbol{a}_{T}=\mathbf{1 0} \mathbf{5}^{\mathbf{5}}$ and various values of $n$ : (a) $n=0.6$, (b) $n=1.0$ and (c) $n=1.4$.

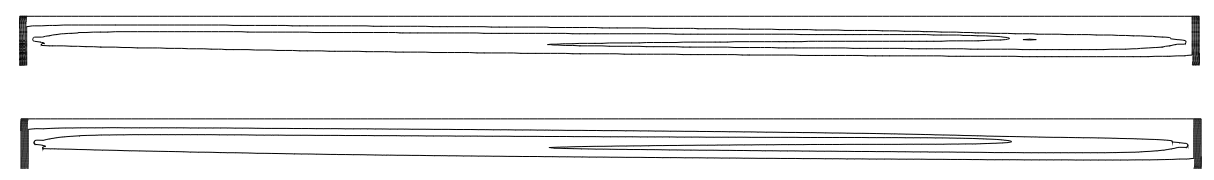

(a)

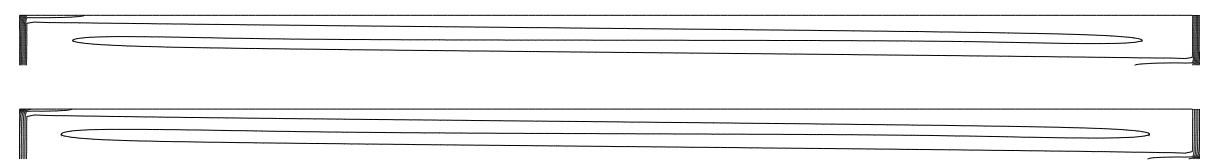

(b)

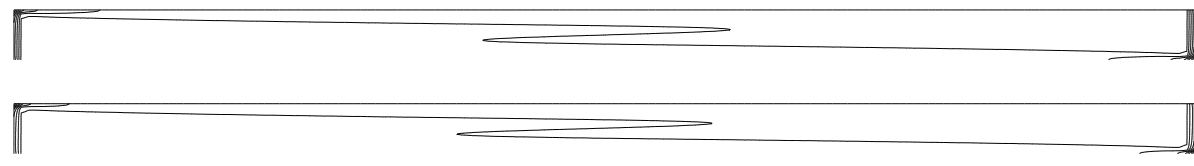

(c)

Fig. 4. Isoconcentrations for $N=-\mathbf{1}$ (top), $N=\mathbf{1}$ (bottom), $\boldsymbol{A}=\mathbf{2 4}, \boldsymbol{L e}=\mathbf{1 0 0}, \boldsymbol{R} \boldsymbol{a}_{\boldsymbol{T}}=\mathbf{1 0} \mathbf{5}^{\mathbf{5}}$ and various values of $n$ : (a) $n=\mathbf{0 . 6}$, (b) $\boldsymbol{n}=\mathbf{1 . 0}$ and (c) $\boldsymbol{n}=\mathbf{1 . 4}$. 

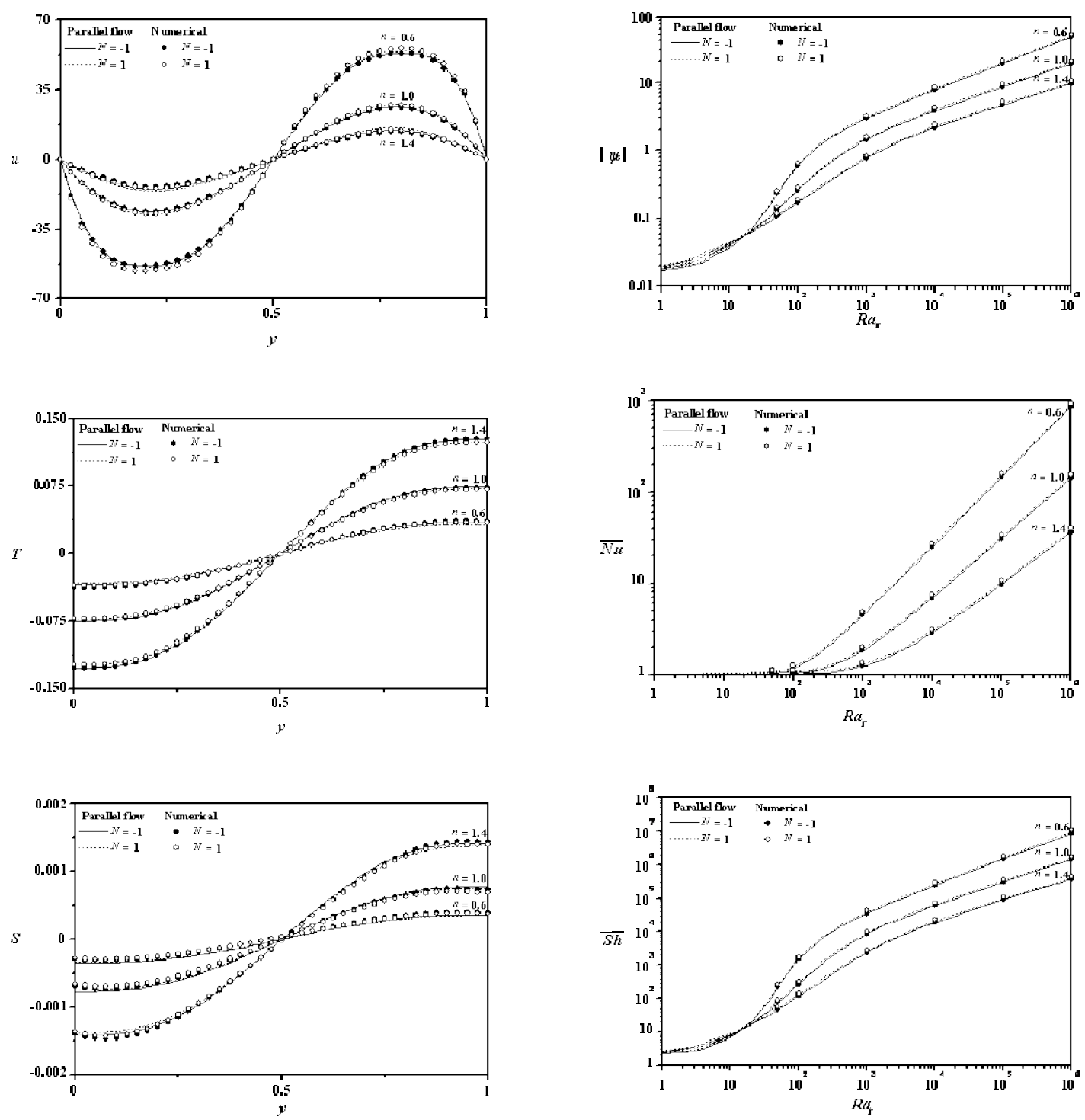

Fig. 5. Horizontal velocity (top), temperature (middle) and concentration (bottom) profiles along the vertical coordinate for $\boldsymbol{A}=\mathbf{2 4}$,

$\boldsymbol{L e}=\mathbf{1 0 0}, \boldsymbol{R} \boldsymbol{a}_{T}=10^{\mathbf{5}}$ and different values of $n$.

Fig. 6. Evolutions of the stream function in the centre of the cavity (top), the average Nusselt number (middle) and the average Sherwood number (bottom) with the Rayleigh number for $\boldsymbol{A}=\mathbf{2 4}, \boldsymbol{L} \boldsymbol{e}=\mathbf{1 0 0}$ and various values of $n$. 